\title{
Adapting a community health facility into an acute care facility during a pandemic
}

\author{
Chong Yau Ong ${ }^{1}$, Lian Leng Low ${ }^{1,2,3}$, Elena bte Mohd Ayob ${ }^{4}$, Jong-Chie Claudia Tien $^{5}$ \\ ${ }^{1}$ Post-Acute Continuity Care, SingHealth Community Hospitals, Singapore, Singapore; ${ }^{2}$ SingHealth-Duke NUS Family Medicine Academic \\ Clinical Programme, Singapore, Singapore; ${ }^{3}$ Family Medicine Continuing Care, Singapore General Hospital, Singapore, Singapore; ${ }^{4}$ Nursing \\ Division, Singapore General Hospital, Singapore, Singapore; ${ }^{5}$ Department of Surgical Intensive Care, Division of Anaesthesiology and Perioperative \\ Medicine, Singapore General Hospital, Singapore, Singapore \\ Correspondence to: Chong Yau Ong, MBBS, MRCP. Outram Community Hospital, 10 Hospital Boulevard, Singapore 168582. \\ Email: ong.chong.yau@singhealth.com.sg.
}

Received: 27 August 2020. Accepted: 22 September 2020; Published: 25 December 2020.

doi: $10.21037 /$ jhmhp-20-109

View this article at: http://dx.doi.org/10.21037/jhmhp-20-109

The COVID-19 pandemic overwhelmed health care systems worldwide, especially in acute care settings. In tandem with community mitigating measures, increasing the capacity in hospitals is of paramount importance. This necessitated the conversion of alternate care facilities to cope with the acute care load (COVID or non-COVID). One potential source of acute hospital beds are community care facilities where much of the healthcare infrastructure is already in place (1-3).

Singapore reported its first confirmed case of COVID-19 on January 23, 2020 and subsequently saw a spike in the number of COVID cases in the population over the following months. National Alert Level was raised on February 7, 2020, indicating that the disease is severe and spreads easily from person to person. As of writing, the number of COVID-19 cases in Singapore had surpassed 50 000 for a population of 6 million.

Singapore General Hospital (SGH) is the largest academic tertiary hospital facility in Singapore with 1,773 beds and one million patient visits annually. Despite having accumulated experience in the SARS outbreak in 2003, the emergence of COVID-19 seventeen years later was a challenge to our resources. The impact of segregating acute hospitals into acute respiratory illness (ARI) wards and non-ARI wards led to tremendous stress on space management. Despite having the highest rates of discharges rates in Singapore, the SGH was also having the highest rates of admissions. A contingency plan was executed immediately to cushion the impact of COVID-19 on our acute care hospital, with an aim to minimise impact on standard patient care. The Outram Community Hospital is a 468-bed-community facility which started operations on November 18, 2019 and was built to provide for step down care from our acute hospital. At the start of the pandemic, the building was not yet fully occupied and the urgent decision was made to mobilize the non-COVID wards from the acute hospital to the community hospital. This decision created a potential increase in hospital beds of more than $20 \%$. The two institutions are connected via a link bridge 350 metres in length.

We describe the strategies of preparation, substitution, adaptation, conservation, reuse, and reallocation of a community hospital to serve patients from an acute hospital setting as part of the surge capacity response to the unprecedented demands during this pandemic.

\section{Space}

Prior to the mobilization, the intended space was inspected for compatibility for use. As a community facility, not all of the bed spaces were constructed with oxygen panels. The building commission was activated for piping works and within a week, all of the beds had been equipped with an oxygen panel and a suction point. The nursing team inspected the wards before operation and checked the functionality of the oxygen points.

As the general hospital and community facility use different telecommunication systems, the support 


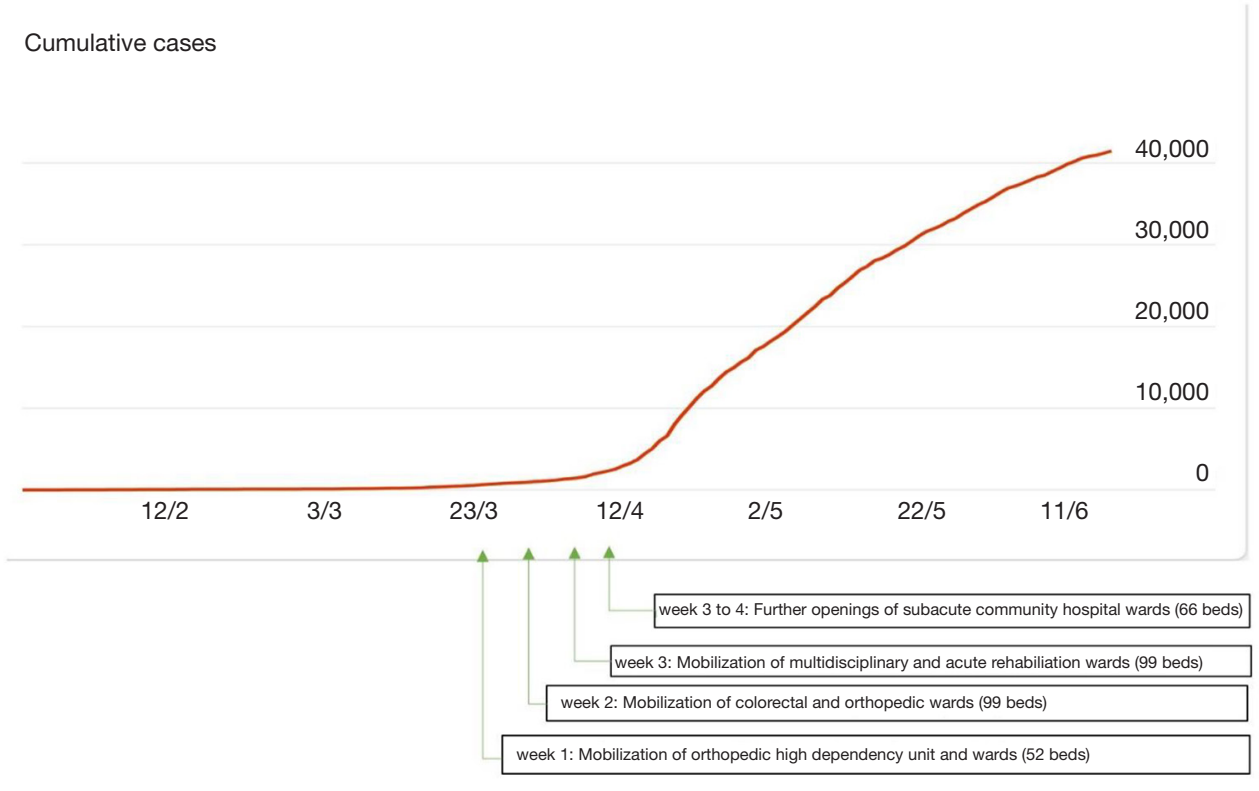

Figure 1 Mobilization of wards from acute hospital into community facility, further ramp-up of community hospital beds, and cumulative number of cases in Singapore.

operations fixed the phone numbers of mobilized wards so as these remained the same and the speed dial to services in the acute hospital were not affected.

Work for pneumatic tube system was accelerated and problems troubleshot. The system became fully functional just before the mobilization of first ward from the acute hospital so that biological specimens could reach the laboratory timely. Prior to that, the porters would have to walk for 20 minutes to transport the specimens to the laboratory building.

The community facility also did not have an automated medication dispensing area due to the planned lower acuity of patients and treatment rooms had to be converted for this purpose.

Because of the mobilization of various teams into the facility, there was an increased need for on-call rooms so that medical personnel could rest during overnight duties. Tutorial rooms were initially converted into on call rooms but were found to be inadequate in terms of ventilation and access to showering facilities. Single bedded isolation rooms were temporarily converted into on-call rooms for the doctors.

This movement into the new space occurred in stages of one-week interval; and by the fourth week, eight wards had been relocated to this facility (Figure 1). Clear signages were present along the corridor and lift lobby exits and temporary signages were added to better direct the new occupants. Frequent inspections of the building safety and compliance with infection prevention control standards were conducted from time-to-time.

The lifts, lift-waiting areas, activity areas and pantries were marked out to ensure compliance with safe social distancing measures.

\section{Staff}

At baseline, there are code blue team present in the community hospital to attend to sudden cardiac arrests. As more medically complex patients were moved to the community facility, the risk of an acute medical emergency was judged to be higher. The code blue workgroup modified the composition of code blue on-duty team from a threeperson-team into a five-person-team. This ensured that the code teams arrived at the code scene in the shortest time possible to initiate acute cardiac life support (ACLS) while the first responders continued basic cardiac life support (BCLS).

The representatives from departments mobilizing to the new facility was engaged to allocate resources for the code blue team. The manpower for each discipline was organized and meetings were held to reach an agreement on the contribution of each discipline based on their functional manpower. As the COVID-19 load in Singapore increased, 
significant manpower had to be deployed to help in the fever screening areas in the emergency department and other COVID-19 related facilities in the communities (4). Many departments were facing dynamic changes in day-today manpower and multiple changes to the daily roster had become a new norm. Teams were briefed about vigilance while holding the code blue phones, responding to test code phone testing, and the workflow of handing over to the next persons on duty. The code blue call centre was informed of the changes of composition of new members and the updated rosters. New members were also briefed on the workflow of the building and orientated to the new building.

Validity of Advanced Cardiac Life Support (ACLS) certification for the newly joined members of the code teams is important. Physicians who had expired or nearexpiry ACLS certification were quickly registered for the next available training slots and an arrangement was made with the hospital management for extra sessions to accommodate these new members. Likewise, nursing staffs were checked for their validity in Life Support Courses for nurses certifications.

Staff were also briefed on infection control and preventive measures so that the practices are in line with international and institutional guidelines. For instance, training for the different Powered Air-Purifying Respirator in the community facility was held. Although the community facility was initially designated for non-COVID patients decanted from the acute hospital, it became more difficult to maintain this demarcation as community spread increased in tandem with the jump in the disease prevalence. In addition, cardiopulmonary resuscitation, intubation and bag mask ventilation are all considered as aerosol generating procedures (5). During the staff movement process, each discipline was also segregated to allow for contact tracing in the event of an outbreak. Additional fire safety drills were conducted for staff who transferred to the community hospital.

\section{Supplies}

With the setting up of new wards in community facilities and the global disruption in supply chain, supplies and equipment were anticipated to be in short supply. We could not rely entirely on traditional procurement processes and had to both conserve current supplies and look for alternative sources. Medically-necessary, time-sensitive surgeries were postponed to minimise potential transmission and to reduce usage of personal protective equipment
(PPE) (6). This move also enabled other products such medications, blood products and antibiotics to be conserved for existing and incoming inpatients.

Some procedural workflows were also amended so as to streamline the manpower required. Innovations such as the creation of SG SAFE, a transparent swabbing booth that comes fitted with a pair of biosafety level 3 gloves, for COVID-19 swabbing came timely as it allowed for conservation of PPE (7). SARS-CoV-2 Real Time PCR (Roche COBAS using Roche 6800 system) was done on all patients prior to the transfer to community facility.

Ensuring that equipment for transfer back to the general hospital in the event of an acute deterioration requiring critical care is necessary. This includes transport ventilators, syringe pumps, oxygen cylinders and a prescribed transfer route.

\section{Standards}

Most healthcare systems can accommodate an 20\% increase in resource requirements without a drop in healthcare standards. Standards of care begin to be compromised when the surge exceeds $100 \%$ of normal capacity (8).

In view of the mobilization of staff into new environments and new medical graduates joining the workforce, supervisors strove to actively train and empower the new members of staff. Induction training was organized such that staff were familiar and comfortable with the newly adopted workflows as well as the new workplace. The support, information, and training were given not only to medical personnel but also to allied health personnel and support operations (9). Electronic platforms were used to minimize face to face contact for these training sessions.

Continuous education about the pandemic and updates on practice guidelines, were disseminated daily to all staff via email. This kept the staff well informed on the latest recommendations and practice by the institution and also helped to reduce staff anxiety.

\section{Conclusions}

The use of a community health facility for acute care is something that needs to be considered during a pandemic. As community health facilities already have much of the infrastructure and manpower for healthcare provision in situ, this provides a good avenue for absorbing some of the increased acute care resources required during a pandemic. Careful planning with rapid execution and constant checks 
are integral to developing and maintaining an agile and proficient system.

\section{Acknowledgments}

Funding: None.

\section{Footnote}

Provenance and Peer Review: This article was a standard submission to the journal. The article did not undergo external peer review.

Conflicts of Interest: All authors have completed the ICMJE uniform disclosure form (available at http://dx.doi. org/10.21037/jhmhp-20-109). The authors have no conflicts of interest to declare.

Ethical Statement: The authors are accountable for all aspects of the work in ensuring that questions related to the accuracy or integrity of any part of the work are appropriately investigated and resolved.

Open Access Statement: This is an Open Access article distributed in accordance with the Creative Commons Attribution-NonCommercial-NoDerivs 4.0 International License (CC BY-NC-ND 4.0), which permits the noncommercial replication and distribution of the article with the strict proviso that no changes or edits are made and the original work is properly cited (including links to both the formal publication through the relevant DOI and the license). See: https://creativecommons.org/licenses/by-nc-nd/4.0/.

\section{References}

1. National Institute of Health and Care Excellence.

doi: 10.21037/jhmhp-20-109

Cite this article as: Ong CY, Low LL, Ayob EBM, Tien JC. Adapting a community health facility into an acute care facility during a pandemic. J Hosp Manag Health Policy 2020;4:40.
Escalation measures: Emergency and acute medical care in over 16s: service delivery and organisation. 2018.

2. Eastman AL, Rinnert KJ, Nemeth IR, et al. Alternate site surge capacity in times of public health disaster maintains trauma center and emergency department integrity: Hurricane Katrina. J Trauma 2007;63:253-7.

3. Jen HC, Shew SB, Atkinson JB, et al. Creation of inpatient capacity during a major hospital relocation: lessons for disaster planning. Arch Surg 2009;144:859-64.

4. Lee WC, Ong CY. Overview of rapid mitigating strategies in Singapore during COVID-19 pandemic. Public Health 2020;185:15-17.

5. Wax RS, Christian MD. Practical recommendations for critical care and anesthesiology teams caring for novel coronavirus (2019-nCoV) patients. Can J Anaesth 2020;67:568-76.

6. World Health Organization. Rational use of personal protective equipment for coronavirus disease (COVID-19) and considerations during severe shortages: interim guidance- 6 April 2020. Available online: https://www. who.int/publications/i/item/rational-use-of-personalprotective-equipment-for-coronavirus-disease-(covid-19)and-considerations-during-severe-shortages

7. The Strait Times. Coronavirus: SGH doctors come up with safer, quicker ways for large-scale testing -18 April 2020. Available online: https://www.straitstimes. com/singapore/health/sgh-doctors-come-up-with-saferquicker-ways-for-large-scale-testing

8. Christian MD, Devereaux AV, Dichter JR, et al. Introduction and executive summary: care of the critically ill and injured during pandemics and disasters: CHEST consensus statement. Chest 2014;146:8S-34S.

9. Hick JL, Hanfling D, Wynia MK, Pavia AT. Duty to plan: health care, crisis standards of care, and novel coronavirus SARS-CoV-2. NAM Perspectives. 2020. 